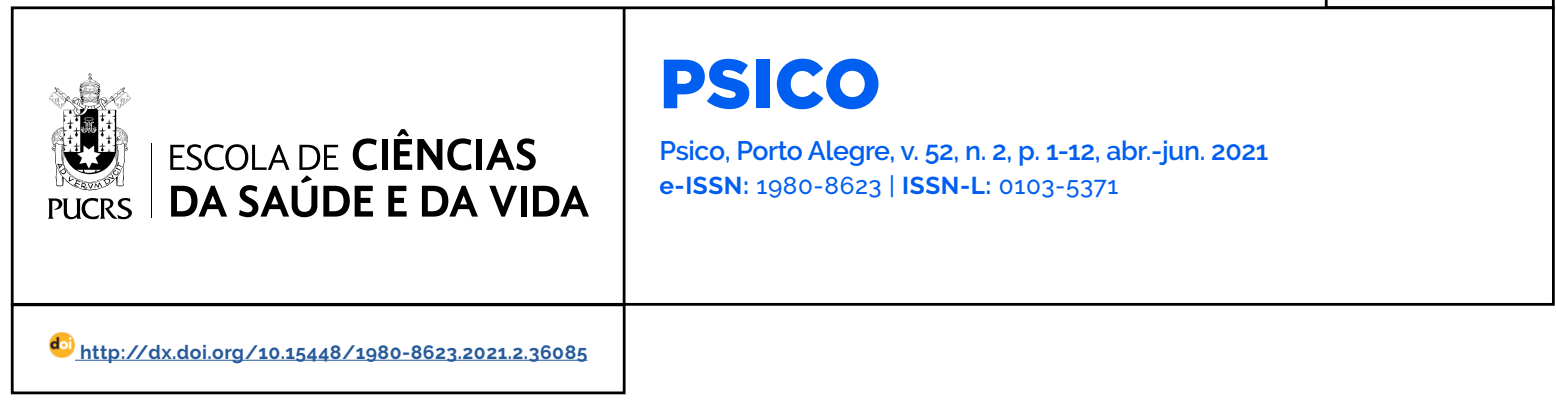

\title{
Estresse ocupacional, Burnout e cultura de segurança do paciente em unidades de perioperatório
}

\author{
Occupational stress, Burnout and patient safety culture in perioperative units \\ Estrés ocupacional, Burnout y cultura de seguridad del paciente en unidades perioperativas
}

\author{
Oclaris Lopes Munhoz ${ }^{1}$ \\ orcid.org/0000-0001-8901-7148 \\ oclaris_munhoz@hotmail.com
}

Laura Prestes Moreira ${ }^{1}$

orcid.org/0000-0002-2577-0832

prestesmoreirag6@gmail.com

\section{Adriane Cristina Bernat \\ Kolankiewicz ${ }^{2}$ \\ orcid.org/0000-0003-1793-7783 \\ adri.saudecoletiva@gmail.com}

\section{Graziele de Lima \\ Dalmolin ${ }^{1}$}

orcid.org/0000-0003-0985-5788

grazi.dalmolin@gmail.com

\section{Tânia Solange Bosi de}

Souza Magnago ${ }^{1}$

orcid.org/0000-0002-5308-1604

tmagnago@terra.com.br

Rafaela Andolhe ${ }^{1}$

orcid.org/0000-0003-3000-8188

rafaela.andolhe@ufsm.br

Recebido em: 22/10/2019

Aprovado em: 09/09/2020.

Publicado em: 10/08/2021.

\section{(c) (i)}

Artigo está licenciado sob forma de uma licença Creative Commons Atribuição 4.0 Internacional.
Resumo: Objetivou-se avaliar a relação entre estresse ocupacional, Burnout e cultura de segurança de profissionais da saúde de unidades de perioperatório. Estudo transversal, desenvolvido com profissionais de saúde de um hospital escola do Rio Grande do Sul, Brasil. Utilizou-se um questionário contendo caracteristicas biossociais e do trabalho; o Safety Attitudes Questionnaire, a Job Stress Scale e o Inventário Maslach de Burnout. Empregou-se análise estatística descritiva e inferencial. Participaram 146 profissionais de saúde que avaliaram negativamente a cultura de segurança do paciente (média=63,8). Prevaleceram profissionais em trabalho de alta exigência $(40,4 \%)$, com alto desgaste emocional (34,9\%), alta despersonalização (44,5\%) e alta realização profissional $(40,4 \%)$. Observou-se correlação baixa e negativa entre despersonalização $(p=-0,254)$, demanda psicológica $(p=-0,246)$ e percepção da cultura de segurança. Ter realização profissional apresentou correlação baixa e positiva com a cultura de segurança $(p=0,256)$. A ocorrência de estresse ocupacional e Burnout possui correlação inversa e significativa com a cultura de segurança.

Palavras-chave: segurança do paciente, saúde do trabalhador, estresse ocupacional, esgotamento profissional, assistência perioperatória

Abstract: This study aimed to evaluate the relationship between occupational stress, Burnout and safety culture of health professionals in perioperative units. Cross-sectional study, developed with health professionals from a teaching hospital in Rio Grande do Sul, Brazil. A questionnaire containing biosocial and work characteristics was used; the Safety Attitudes Questionnaire, the Job Stress Scale and the Maslach Burnout Inventory. Descriptive and inferential statistical analysis was used. 146 health professionals participated in the study, who negatively assessed the patient's safety culture (mean $=63.8$ ). Professionals predominant in high demand work (40.4\%), with high emotional exhaustion (34.9\%), high depersonalization (44.5\%) and high professional performance (40.4\%). There was a low and negative correlation between depersonalization ( $p=-0.254)$, psychological demand $(p=-0.246$ ) and the perception of safety culture. Having professional accomplishments had a low and positive correlation with safety culture $(p=0.256)$. The occurrence of occupational stress and burnout has a significant inverse correlation with the safety culture.

Keywords: patient safety, occupational health, occupational stress, burnout professional, perioperative care

Resumen: Este estudio tuvo como objetivo evaluar la relación entre el estrés laboral, el Burnout y la cultura de seguridad de los profesionales de la salud en las unidades perioperatorias. Estudio transversal, desarrollado con profesionales de la salud de un hospital universitario en Rio Grande do Sul, Brasil. Se utilizó un cuestionario con caracteristicas biosociales y laborales; el cuestionario de actitudes de seguridad, la escala de estrés laboral y el inventario de agotamiento de Maslach. Se utilizó un análisis estadístico descriptivo e inferencial. En el estudio participaron 146 profesionales de la salud, quienes valoraron negativamente la cultura de seguridad del paciente (media $=63,8$ ). Profesionales predominantes en trabajos de alta demanda (40.4\%), con alto agotamiento emocional (34.9\%), alta 
despersonalización (44.5\%) y alto desempeño profesional (40.4\%). Hubo una correlación baja y negativa entre la despersonalización $(p=-0.254)$, la demanda psicológica ( $p=-0.246$ ) y la percepción de la cultura de seguridad. Tener logros profesionales tuvo una correlación baja y positiva con la cultura de seguridad $(p=0.256)$. La aparición de estrés laboral y agotamiento tiene una correlación inversa significativa con la cultura de seguridad.

Palabras clave: seguridad del paciente, salud laboral, estrés laboral, agotamiento profesional, atención perioperativa

Um dos agravos à saúde do trabalhador que mais atinge os profissionais de saúde é o estresse, que pode estar associado a um conjunto de forças externas que resultam em efeitos permanentes ou transitórios sobre o individuo (Oliveira et al., 2013). No que concerne ao estresse ocupacional, pode estar relacionado a altas demandas psicológicas, ao baixo controle sobre como fazer o seu trabalho e ao baixo apoio social recebido de colegas e da chefia no ambiente laboral (Karasek \& Theörel, 1990). Ele causa desgaste e pode influenciar na atuação da equipe durante a prestação da assistência, aspecto propulsor para a insegurança (Andrade \& Costa, 2014; Karasek \& Theörel, 1990).

Nesse sentido, um dos referenciais teórico-metodológicos que avalia o estresse ocupacional é - Modelo Demanda-Controle (MDC), que busca elucidar as exigências mentais que implicam no trabalho profissional. Ainda, pauta-se em pressupostos, a exemplo: altas demandas psicológicas e baixo controle acerca do processo de trabalho (trabalho de alta exigência) resultam em reações adversas à saúde do profissional; se existem alta demanda psicológica e alto controle (trabalho ativo), simultaneamente, há um "efeito favorável" do estresse; por outro lado, quando há baixa demanda e baixo controle (trabalho passivo), resulta em redução de habilidades e desmotivação por parte do profissional (Karasek \& Theörel, 1990). Em contraponto, o trabalho em que há baixa demanda e alto controle (trabalho de baixa exigência) traduz uma situação ideal e favorável para a saúde do trabalhador (Karasek \& Theörel, 1990).

Assim sendo, sabe-se que os profissionais de saúde executam atividades de atendimento individual e coletivo, vivenciam relacionamentos interpessoais e multiprofissional os quais, muitas vezes, são conflitantes (Silva \& Moura, 2014). Soma- -se a isso a falta de recursos materiais e humanos, especialmente nos hospitais públicos, o que pode resultar em carga de trabalho excessiva, elevando os riscos de esgotamento e de adoecimento físico e psíquico dos profissionais, bem como da ocorrência de incidentes com os pacientes (Silva \& Moura, 2014).

Em uma situação de incidente envolvendo o paciente, por exemplo, o profissional torna-se uma segunda vítima e, em decorrência, fica traumatizado. Isso repercute em impacto emocional, com perda de reputação, desconfiança por parte dos colegas e chefias, o que pode afetar tanto sua vida profissional, como pessoal. Assim, a instituição tem papel fundamental para buscar tratar essa situação de forma sistêmica e não centrada na pessoa; bem como fornecendo as condições adequadas para o desenvolvimento do trabalho (Romero et al., 2018).

Nesse contexto, no ambiente hospitalar, o cuidado perioperatório exige alto grau de qualificação e responsabilidade dos profissionais atuantes nas unidades cirúrgicas. Nesses locais, tanto há demanda de procedimentos complexos, como a necessidade de lidar com os sentimentos dos pacientes e familiares. Dentre as unidades que prestam assistência aos pacientes com afecções cirúrgicas estão as unidades de internação (pré e pós-operatório), os blocos cirúrgicos (intraoperatório) e as salas de recuperação anestésica (pós-operatório imediato). Assim, percebe-se que essas particularidades exigem que os profissionais de saúde enfrentem adequadamente os estressores laborais (Amthauer \& Falk, 2014; Andolhe et al., 2015; Brandão \& Galvão, 2013; Gonçalves \& Medeiros, 2016).

No entanto, quando não ocorre enfrentamento e o controle do estresse não é efetivo, os profissionais podem desenvolver a sindrome de Burnout, considerada como uma síndrome multidimensional em resposta ao estresse crônico, que gera sentimentos como exaustão, incapacidade, fracasso. Ela está relacionada às consequências do trabalho na saúde dos profissionais e atinge, principalmente, os trabalhadores que atuam como cuidadores, já que atuam em contato direto com os pacientes. Assim, as condições excessivas e estressantes 
do ambiente de trabalho podem refletir na saúde mental e física do trabalhador e, indiretamente, comprometer a segurança do paciente (Gonçalves \& Medeiros, 2016; Maslach, 2009; Souza \& Araujo, 2015). Para tanto, torna-se pertinente avaliar a cultura de segurança do paciente.

Nos últimos anos, tem crescido o número de estudos acerca dos ambientes laborais e o seu impacto sobre a cultura de segurança, que é considerada um "conjunto de valores, atitudes, competências e comportamentos que determinam o comprometimento com a gestão da saúde e da segurança, substituindo a culpa e a punição pela oportunidade de aprender com as falhas e melhorar a atenção à saúde" (Agência Nacional de Vigilância Sanitária [Anvisa], 2013, p. 2). Um dos instrumentos que faz a avaliação e gera indicadores para a segurança do paciente é o Safety Attitudes Questionnaire (SAQ) (Carvalho \& Cassiani, 2012). Também, uma vez mensurado, pode ser considerado como indicador de comportamento, produtividade, motivação e satisfação dos profissionais (Carvalho \& Cassiani, 2012; Corregio et al., 2014).

Com vistas a conhecer os constructos do presente artigo, realizou-se um estudo de revisão na base de dados eletrônica Literatura Latino-Americana e do Caribe em Ciências da Saúde (LILACS) e na Base de Dados Bibliográfica Especializada na Área de enfermagem (BDENF), via Biblioteca Virtual de Saúde (BVS), que teve como estratégia de busca avançada: (["cultura organizacional" OR "administração da segurança" OR "gestão do perigo"] AND ["erro humano" OR "estresse ocupacional" OR "esgotamento profissional"] OR ["cuidados terciários de saúde"]). Não foram encontrados estudos que avaliassem conjuntamente o estresse ocupacional e o Burnout dos profissionais de saúde e suas relações com a cultura de segurança do paciente em unidades de perioperatório, especificadamente. Assim, este estudo pretende contribuir trazendo evidências científicas a respeito da interface entre a saúde do trabalhador, no que tange ao estresse e ao Burnout, e a segurança do paciente.

Com base nessas considerações, delineou-se a seguinte questão de pesquisa: Existe relação entre o estresse ocupacional, o Burnout dos profissionais de saúde e a percepção da cultura de segurança em unidades de perioperatório? Nesse sentido, este estudo objetivou avaliar a relação entre estresse ocupacional, Burnout e cultura de segurança de profissionais da saúde de unidades de perioperatório. A hipótese era de que os trabalhadores com niveis elevados de estresse ocupacional (alta exigência no trabalho) e com Burnout avaliam negativamente a cultura de segurança em unidades de perioperatório.

\section{Método}

\section{Delineamento e cenário do estudo}

Estudo transversal, realizado nas unidades de perioperatório de um hospital de ensino da região central do Rio Grande do Sul, Brasil. Essas unidades correspondem à unidade de cirurgia geral - serviço de internação (UCG-SI), ao bloco cirúrgico $(\mathrm{BC})$ e às salas de recuperação anestésica (SRA) e intermediária (SRI)

\section{Participantes do estudo}

Participaram do estudo: enfermeiros, técnicos e auxiliares de enfermagem, médicos, fisioterapeutas, dentistas, psicólogos, assistentes sociais, fonoaudiólogos, nutricionistas e auxiliares de saúde. Foram incluidos no estudo os profissionais de saúde com no mínimo quatro semanas de atuação e carga horária mínima de 20 horas semanais; e excluidos os profissionais afastados no período da coleta por qualquer motivo.

A seleção dos participantes ocorreu por amostragem de conveniência e não probabilística. De um total de 181 profissionais atuantes nas unidades de perioperatório, participaram 146, correspondendo a $80,7 \%$ da população elegivel para o estudo.

Procedimentos, coleta de dados, instrumentos de pesquisa

A coleta de dados ocorreu no período de março a julho de 2018. Após tramitação ética e autorização institucional, realizou-se um contato prévio com as chefias das unidades de perioperatório, com vistas a informar os objetivos e a convidar os profissionais de saúde para o estudo. Eles foram 
convidados a participar da pesquisa e a preencherem os instrumentos de coleta de dados no local de trabalho, em ambiente reservado, e de maneira que cada um pudesse ausentar-se por alguns minutos das suas atividades. Conforme consentimento assinado por meio do Termo de Consentimento Livre e Esclarecido (TCLE), em duas vias, entregava-se um envelope contendo um instrumento para a caracterização dos profissionais (sexo, idade, estado civil, possuir filhos, setor, regime de trabalho, cargo, maior formação completa, turno de trabalho, vínculo com mais de um emprego, satisfação em trabalhar na unidade e intenção de deixar o emprego no hospital), a Job Stress Scale (JSS) (Alves et al., 2004), o Maslach Burnout Inventory - Human Service Survey (MBI-HSS) (Lautert, 1995) e o Safety Attitudes Questionnaire (SAQ) (Carvalho \& Cassiani, 2012).

Para a avaliação do estresse ocupacional, utilizou-se a JSS, versão reduzida e adaptada para a realidade brasileira com Alpha de Cronbach variando entre 0,63 e 0,86 (Alves et al., 2004). Essa versão possui 17 itens, e é subdividida em três domínios: (i)demanda psicológica, domínio avaliado por cinco questões; (ii) controle sobre o trabalho, representado por seis questões; e (iii) apoio social (não foi utilizado nas análises do presente estudo). Como opções de respostas aos itens de demanda psicológica e controle, utiliza-se uma escala do tipo Likert (1 a 4), que compreende: frequentemente (4), às vezes (3), raramente (2) e nunca (1). Para a dimensão demanda psicológica a pontuação pode variar de 4 até 20 pontos (quanto maior o escore, maior a demanda psicológica) e, para a dimensão controle, a variação pode ocorrer de 4 até 24 pontos (quanto maior o escore, maior o controle). As questões de número 4 e 9 possuem pontuação reversa (Alves et al., 2004).

Para o levantamento do nivel de Burnout, utilizou-se o Maslach Burnout Inventory (IMB), traduzido e com evidências de validade em português, com Alpha de Cronbach de 0,89 (Lautert, 1995; Maslach \& Jackson, 1986). O inventário, possui, atualmente, 22 itens distribuidos nas dimensões desgaste emocional, despersonalização e realização profissional. Para cada questão existe uma escala do tipo Likert que varia de zero (nunca) a 4 (diariamente), onde o participante assinala a frequência com que se percebe ou se sente a respeito do enunciado de cada questão. Altas pontuações em desgaste emocional e despersonalização, associadas à baixa pontuação em realização profissional indicam que o indivíduo está em Burnout (Lautert, 1995; Maslach \& Jackson, 1986).

O SAQ foi utilizado para avaliar a percepção e as atitudes em relação à cultura de segurança do paciente. Esse instrumento que foi traduzido com evidências de validade para a realidade brasileira, apresentando um Alpha de Cronbach de 0,89 (Carvalho \& Cassiani, 2012). É composto por 36 itens, distribuidos em seis domínios: clima de trabalho em equipe, clima de segurança, satisfação no trabalho, percepção do estresse, percepção da gerência da unidade e do hospital e condições de trabalho. Para a resposta a cada questão, os autores indicam uma escala Likert de cinco pontos, variando de: "discordo totalmente" a "concordo totalmente". Os itens 2, 11 e 36 do questionário são questões reversas (Carvalho \& Cassiani, 2012).

\section{Análise dos dados}

A simetria dos dados foi testada por meio do teste de Kolmogorov-Smirnov e apresentou-se assimétrica. Para a dicotomização dos domínios da JSS foi utilizada a mediana, tendo em vista a assimetria dos dados. Conforme a distribuição dos dados, os valores obtidos foram categorizados em quartil a partir de cada dimensão, correspondendo a baixa e alta demanda psicológica (7 - 13 pontos = baixa; 14 - 19 pontos = alta), baixo e alto controle (11 - 18 pontos = baixo; $19-22$ pontos = alto). A partir disso, realizou-se a formação dos quadrantes do MDC em: baixa exigência; trabalho passivo; trabalho ativo; alta exigência. Categoria de referência: baixa exigência.

Para a análise do nível de Burnout, os pontos de corte para as três dimensões foram obtidos por tercis, como recomendado pelo Manual do MBI (Lautert, 1995). Os pontos de corte para a amostra estudada foram: desgaste emocional: baixo $\leq 6$; médio = entre 6,1 e 10,9; alto $\geq 11$; despersonalização: baixa s 1; média = entre 1,1 e 2,9; alta $\geq 3$; realização profissional: baixa $\leq 20$; média 
= entre 20,1 e 23,9; alta $\leq 24$.

Para a análise dos escores dos domínios do $S A Q$, realizou-se a soma das pontuações para cada questão que compõe o domínio, dividida pelo número de itens que compõem o respectivo domínio. Para cada dimensão do instrumento, utilizou-se a fórmula $(m-1) \times 25$, onde $m$ é a média dos itens do domínio em questão. O escore final do instrumento varia de o a 100, no qual zero corresponde à pior percepção de atitudes de segurança pelos profissionais de saúde e 100, à melhor percepção. São considerados valores positivos quando o total do escore é igual ou superior a 75. Ressalta-se que essa análise é própria do instrumento SAQ e é recomendada pelas autoras do constructo (Carvalho \& Cassiani, 2012).

Os dados foram digitados no Excel, com dupla digitação independente. Após conferência de erros e inconsistências da digitação, os dados foram processados pelo Statistical Package for the Social Sciences (SPSS), versão 18.0. As características sociodemográficas e do trabalho foram apresentadas com frequências absolutas e relativas. Para a identificação da associação entre percepção da cultura de segurança, niveis de estresse e Burnout utilizou-se o teste do Qui-Quadrado ou Exato de Fischer. Para as correlações entre as variáveis quantitativas utilizou-se a correlação de Spearman. Considerou-se valor de $|r|=1$ uma correlação perfeita; $0,80 \leq|r|<1$, correlação muito alta; $0,60 \leq|r|<0,80$, correlação alta; $0,40 \leq|r|<0,60$, correlação moderada; 0,20 $|r|<0,40$, correlação baixa; $0<|r|<0,20$, correlação muito baixa; e r=0, correlação nula (Prieto \& Muñiz, 2000). As associações foram consideradas estatisticamente significantes quando o valor de $\mathrm{p}$ foi menor que 0,05

\section{Aspectos éticos}

Esta pesquisa seguiu todos os preceitos éticos que envolvem estudos com seres humanos, ten- do aprovação do Comitê de Ética em Pesquisa da Universidade Federal de Santa Maria em dezembro de 2017, sob o Parecer n 2.447.277 e CAAE: 80587417.0.0000.5346.

\section{Resultados}

Participaram do estudo 146 (80,7\%) profissionais de saúde, sendo 55 (38\%) lotados na UCG-SI, outros 55 (38 \%) no BC e 55 (38\%) na SRA e na SRI. Destes, 74 (50,7\%) eram técnicos de enfermagem, 30 (20,5 \%) eram enfermeiros, 21 (14,4 \%) eram médicos, 10 (6,8 \%) eram auxiliares de enfermagem e 11 (7,6 \%) corresponderam a outros (assistente social, fisioterapeuta, fonoaudiólogo, nutricionista e auxiliar de saúde). Optou-se por categorizar os demais profissionais dessa forma para não haver o risco de identificação, visto que o quantitativo de profissionais nessas categorias foi menor. A mediana de idade dos profissionais foi de 41,1 anos (mínimo de 20, máximo de 60 anos).

Houve maior percentual dos profissionais correspondente ao sexo feminino ( $n=104 ; 71,2 \%$ ), com companheiro ( $n=112 ; 76,7 \%$ ) e com filhos ( $n=100$; $68,5 \%$ ). A maior formação prevalente foi o ensino médio ( $n=50 ; 34,2 \%$ ), seguida de especialização/ residência $(n=45 ; 30,8 \%)$.

No que se refere às características do trabalho, maior percentual dos profissionais de saúde atuava no turno da noite ( $n=44 ; 30,2 \%)$, pertencente ao regime de trabalho celetista ( $n=85 ; 58,2 \%$ ), sem outro vínculo empregatício ( $n=125 ; 85,6 \%$ ), e, daqueles que possuiam outro vínculo $(n=21 ; 14,4$ $\%$ ), prevaleceram os que trabalham na assistência em saúde ( $n=11 ; 52,4 \%$ ). Ainda, eram maioria os profissionais que estavam satisfeitos em seus setores ( $n=137 ; 94.5 \%$ ) e não tinham intenção de deixar o serviço ( $n=114 ; 89,8 \%$ ).

Na Tabela 1, está a frequência dos profissionais de saúde segundo os quadrantes do MDC e as dimensões de Burnout. 
Tabela 1 - Distribuição dos profissionais de saúde segundo os quadrantes do Modelo Demanda-Controle e as dimensões de Burnout

\begin{tabular}{|c|c|c|c|c|}
\hline $\begin{array}{l}\text { Quadrante Modelo } \\
\text { Demanda-Controle }\end{array}$ & Baixa Exigência & $\begin{array}{l}\text { Trabalho } \\
\text { Ativo }\end{array}$ & $\begin{array}{l}\text { Trabalho } \\
\text { Passivo }\end{array}$ & $\begin{array}{c}\text { Alta } \\
\text { Exigência }\end{array}$ \\
\hline & n (\%) & $n(\%)$ & n (\%) & n (\%) \\
\hline \multirow{6}{*}{ Dimensões de Burnout } & $28(19,2)$ & $34(23,3)$ & $23(15,8)$ & $59(40,4)$ \\
\hline & & Baixo & Médio & Alto \\
\hline & & $\mathrm{n}(\%)$ & $\mathrm{n}(\%)$ & n (\%) \\
\hline & Desgaste Emocional ( $n=144)$ & $47(32,2)$ & $46(31,5)$ & $51(34,9)$ \\
\hline & Despersonalização (n=145) & $46(31,5)$ & $34(23,3)$ & $65(44.5)$ \\
\hline & $\begin{array}{l}\text { Realização Profissional } \\
\qquad(n=145)\end{array}$ & $43(29,5)$ & $43(29,5)$ & $59(40,4)$ \\
\hline
\end{tabular}

Conforme Tabela 1, verificou-se prevalência de profissionais de saúde no quadrante de alta exigência ( $n=59 ; 40,4$ \%). Ademais, prevaleceram os profissionais com alto desgaste emocional $(n=51$; $34,9 \%$ ), com alta despersonalização ( $n=65 ; 44.5 \%$ ) e com alta realização profissional ( $n=59 ; 40,4 \%$ ). A Tabela 2 descreve os resultados obtidos acerca da cultura de segurança do paciente na percepção dos profissionais de saúde das unidades de perioperatório.

Tabela 2 - Análise descritiva do Questionário de Atitude de Segurança (SAQ) segundo a percepção dos profissionais de saúde das unidades de perioperatório

\begin{tabular}{|c|c|c|c|c|c|c|c|c|c|}
\hline \multirow{4}{*}{$\begin{array}{c}\begin{array}{c}\text { Dominios do } \\
\text { SAQ }\end{array} \\
\text { SAQ Total }\end{array}$} & \multirow{4}{*}{$\begin{array}{c}\mathbf{N} \\
114\end{array}$} & \multirow{4}{*}{$\begin{array}{c}\text { Média } \\
63,8\end{array}$} & \multirow{4}{*}{$\begin{array}{c}\text { Desvio Padrão } \\
14,0\end{array}$} & \multirow{4}{*}{$\frac{\text { Minimo }}{}$} & \multirow{4}{*}{$\frac{\text { Máximo }}{96,5}$} & \multicolumn{4}{|c|}{ Cultura de Segurança } \\
\hline & & & & & & \multirow{2}{*}{\multicolumn{2}{|c|}{$\frac{\text { Positiva }}{n(\%)}$}} & \multirow{2}{*}{\multicolumn{2}{|c|}{$\frac{\text { Negativa }}{n(\%)}$}} \\
\hline & & & & & & & & & \\
\hline & & & & & & 29 & 19,9 & 85 & 58,2 \\
\hline $\begin{array}{c}\text { Clima de trabalho } \\
\text { em equipe }\end{array}$ & 138 & 67,8 & 17,2 & 16,6 & 100,0 & 57 & 39,0 & 81 & 55.5 \\
\hline $\begin{array}{l}\text { Clima de } \\
\text { segurança }\end{array}$ & 139 & 63,6 & 17,9 & 14,2 & 100,0 & 39 & 26,7 & 100 & 68,9 \\
\hline $\begin{array}{l}\text { Satisfação no } \\
\text { trabalho }\end{array}$ & 141 & 83,0 & 15,2 & 30,0 & 100,0 & 111 & 76,0 & 30 & 20,5 \\
\hline $\begin{array}{l}\text { Percepção de } \\
\text { estresse }\end{array}$ & 137 & 67,6 & 25,9 & 0,0 & 100,0 & 76 & 52,1 & 61 & 41,8 \\
\hline $\begin{array}{l}\text { Percepção da ge- } \\
\text { rência da unidade }\end{array}$ & 136 & 55,2 & 20,3 & 4,1 & 100,0 & 27 & 18,5 & 109 & 74,7 \\
\hline $\begin{array}{l}\text { Percepção da ge- } \\
\text { rência do hospital }\end{array}$ & 136 & 47,6 & 22,0 & 0,0 & 100,0 & 20 & 13,7 & 116 & 79,5 \\
\hline $\begin{array}{c}\text { Condições de } \\
\text { trabalho }\end{array}$ & 143 & 61,0 & 24,3 & 8,3 & 100,0 & 62 & 42,5 & 81 & 55,5 \\
\hline
\end{tabular}


Segundo a análise do $S A Q$, os profissionais de saúde das unidades de perioperatório tinham uma percepção negativa acerca da cultura de segurança (média = 63,8). Quanto aos domínios, somente o domínio de satisfação no trabalho obteve percepção positiva (média $=83,0$ ).

A Tabela 3 apresenta a correlação entre estres- se ocupacional, Burnout e cultura de segurança.

Conforme a Tabela 3, observa-se correlação baixa e negativa entre as seguintes variáveis: despersonalização, demanda psicológica e percepção da cultura de segurança do paciente. A variável realização profissional apresentou correlação baixa e positiva com a cultura de segurança.

Tabela 3 - Correlação de entre estresse ocupacional, Burnout e cultura de segurança

\begin{tabular}{|c|c|c|c|c|c|}
\hline Variáveis & $\begin{array}{l}\text { SAQ } \\
\text { Geral }\end{array}$ & $\begin{array}{l}\text { Desgaste } \\
\text { Emocional }\end{array}$ & $\begin{array}{l}\text { Desperso- } \\
\text { nalização }\end{array}$ & $\begin{array}{l}\text { Realização } \\
\text { Profissional }\end{array}$ & $\begin{array}{l}\text { Demanda } \\
\text { Psicológica }\end{array}$ \\
\hline 1. SAQ Geral & 1 & & & & \\
\hline 2. Desgaste Emocional & -.389 & 1 & & & \\
\hline 3. Despersonalização &,- 254 & .597 & 1 & & \\
\hline 4. Realização Profissional &, $256^{\prime \prime}$ &,- 332 &,- 331 & 1 & \\
\hline 5. Demanda Psicológica &,$- 246 "$ & $491^{\prime \prime}$ & $.366^{\prime \prime}$ &,- 155 & 1 \\
\hline 6. Controle sobre o Trabalho &, 175 & .066 & ,139 & 139 &,- 151 \\
\hline
\end{tabular}

${ }^{*}$ Correlação é significativa no nivel 0,01 (bicaudal) - Correlação de Spearman.

\section{Discussão}

No que se refere ao estresse ocupacional, prevaleceram os profissionais de saúde com alta exigência no trabalho, ou seja, com altas demandas psicológicas e baixo controle sobre o trabalho. Essa situação indica que os participantes estavam com sua saúde em risco como resultado das atividades laborais desenvolvidas nas unidades de perioperatório. Somados a isso, fatores como o desgaste emocional, o cansaço e a ansiedade, se vivenciados pelos profissionais, podem predispô-los ao adoecimento relacionado ao trabalho (Andolhe et al., 2015; Andrade \& Costa, 2014; Munhoz et al., 2020).

Depreende-se que a alta exigência evidenciada nas unidades de perioperatório desta pesquisa, além de relacionar-se com a demanda de trabalho manual/procedimentos assistenciais, também está associada às exigências psicológicas a que os profissionais de saúde estão sujeitos. A exemplo disto, sabe-se que os pacientes que são submetidos a cirurgias relatam ter angústias, medo de não sobreviver e até mesmo desconhecem a forma como será realizado determinado procedimento, o que requer que os profissionais realizem orientações quanto aos procedimentos, buscando acalmá-los e confortá-los (Gonçalves \& Medeiros, 2016; Munhoz et al., 2018). Essa situação caracteriza-se como um estressor laboral e exige dos profissionais preparo emocional, ou seja, para que se tenha um melhor controle sobre o trabalho.

Somado a isso, considerando os ambientes de perioperatório deste estudo, outro fator relacionado ao estresse ocupacional são as cirurgias não programadas, as quais ocorrem, predominantemente, no periodo noturno, no qual não há cirurgias agendadas na instituição. Levando em conta que a prevalência do turno de trabalho evidenciada nesta pesquisa é de profissionais atuantes no noturno, é possivel que os trabalhadores do bloco cirúrgico enfrentem maior estresse, pois as cirurgias de emergência exigem preparo e agilidade em um curto periodo de tempo (Boas et al., 2016).

Também, é importante considerar a prevalência de profissionais atuantes no turno da manhã, visto que, além de a unidade de clinica cirúrgica ser a maior unidade de internação da instituição, é no referido turno que se concentra o maior número de procedimentos, demandando alta carga 
de trabalho dos profissionais. Esses fatores corroboram a alta exigência evidenciada no estudo.

Chama a atenção o fato de, dos 146 profissionais, 34 se encontrarem em trabalho ativo, pois é neste contexto em que o trabalho é enfrentado como um desafio, podendo proporcionar crescimento e aprendizado pessoal e profissional. Ainda, outros 23 profissionais estavam trabalhando com ausência de desafios e iniciativas (trabalho passivo), o que pode resultar em declínio gradual de aprendizagem e desenvolvimento (Alvez et al., 2004; Munhoz et al., 2020; Karasek \& Theörell, 1990).

No que se relaciona com a avaliação individual das dimensões do IMB, se sobressaíram os profissionais de saúde com alto desgaste emocional, despersonalização e realização profissional. Assim, evidencia-se que a maioria dos investigados se sentia sem energia para trabalhar e com perda de entusiasmo e interesse pessoal, mas, mesmo assim, gostavam do que faziam. Na perspectiva socioambiental do Burnout, percebe-se que as estratégias de enfrentamento utilizadas pelos mesmos podem não ser efetivas para o enfrentamento do estresse.

Os achados acima divergem dos encontrados por pesquisa que buscou avaliar a sindrome de Burnout em profissionais da área da saúde atuantes em Minas Gerais, na qual constataram-se niveis médios para as três dimensões de Burnout (Carvalho et al., 2016). Os autores destacaram que cinco profissionais da amostra estudada estavam com altos niveis de desgaste emocional e despersonalização e outros 47, com niveis altos de despersonalização, resultados que demonstram que os profissionais apresentaram algum tipo de sofrimento (Carvalho et al., 2016). Nesse contexto, embora a ciência em saúde tenha avançado, proporcionando conhecimentos técnicos científicos de novas formas para combater os estressores laborais, percebe-se que o Burnout tem crescido em maiores proporções do que de possibilidades de enfrentamento.

Destarte, Zanatta \& Lucca (2015) constataram niveis médios para as dimensões do IMB, resultado que vai de encontro aos achados nesta investigação, pois, na análise das dimensões, muitos dos participantes atenderam ao critério de duas dimensões, ou seja, estavam sujeitos ao Burnout. Ainda, os autores evidenciaram que, dentre as categorias profissionais, os médicos apresentaram maiores prevalências para alto desgaste emocional e para sentimentos de baixa realização profissional, enquanto para os enfermeiros se destacou o alto nivel de despersonalização (Zanatta \& Lucca, 2015). Depreende-se que o esgotamento profissional ocasionado pela síndrome seja impactante para a cultura de segurança, pois faz com que a percepção de possibilidade de atuação por parte dos indivíduos seja diminuida, ocasionando possiveis erros que não deveriam acontecer.

Reflete-se sobre os ambientes de perioperatório e sua relação com o Burnout, pois, mesmo com avanços na perspectiva da saúde do trabalhador, percebe-se que esses cenários interferem na saúde ocupacional e emocional dos profissionais. Essas circunstâncias podem estar relacionadas com o perfil de pacientes atendidos pelos profissionais de saúde, uma vez que em todos os cenários estudados, inclusive na unidade de internação (em menor proporção), há pacientes críticos, com um ou mais sistemas fisiológicos comprometidos, geralmente sedados e em uso de ventilação mecânica. Ou seja, além da capacidade técnica e científica, exige que os profissionais tenham preparo emocional para atender esses pacientes, o que reflete em um indivíduo desgastado emocionalmente e despersonalizado, indo ao encontro do evidenciado na amostra estudada (Alves et al., 2004; Andolhe et al., 2015; Lautert, 1995).

No que concerne à cultura de segurança, os profissionais de saúde das unidades de perioperatório demonstraram uma avaliação negativa do clima de segurança nesses ambientes. Ainda, conforme o panorama dos domínios do $S A Q$, unicamente o referente à satisfação no trabalho obteve percepção positiva. Pesquisa que objetivou avaliar a cultura de segurança em três hospitais públicos do Brasil, na análise geral do SAQ, também evidenciou um clima negativo das três instituições, com médias entre 65 e 69 . Esse mesmo estudo constatou que dois dos três hospitais tinham percepção positiva para o do- 
minio clima de trabalho em equipe (média=75) e os três hospitais tinham percepção positiva para o domínio satisfação no trabalho, com médias de 80, 81 e 83, e os demais domínios obtiveram percepção negativa (<75) (Carvalho et al., 2017). Nesse sentido, a satisfação profissional está relacionada a um bom relacionamento interpessoal, diálogo aberto e a ter suporte quando necessário. Assim, as ações desenvolvidas pelos profissionais e a segurança do paciente são favorecidas (Carvalho et al., 2017; Matiello et al, 2016).

Quanto aos domínios percepção da gerência da unidade e do hospital, esses foram os que obtiveram os piores escores, com médias de 55,2 e 47,6, respectivamente. Dados que corroboram outras investigações em que se evidenciou semelhante resultado (Carvalho et al., 2017; Matiello et al., 2016). Essa realidade pode refletir a forma como as gerências institucionais desenvolvem suas ações, pois entende-se que são precursoras de boas atitudes. Depreende-se que, ao haver horizontalidade e diálogo aberto entre gestores e assistentes, haverá um bom relacionamento interpessoal e a interface saúde do trabalhador e segurança do paciente será favorecida.

Outros domínios avaliados negativamente foram clima de trabalho em equipe e de segurança, que envolvem a percepção de aspectos relacionados a como problemas no cuidado ao paciente são tratados, sobre apoio da equipe, comunicação, sentir-se seguro se fosse paciente na unidade, como erros são tratados, entre outros. Assim, evidencia-se que essses fatores estavam fragilizados nas unidades de perioperatório e que os profissionais não se sentiriam seguros se fossem pacientes nesses ambientes. Esse resultado pode indicar que os profissionais percebiam o ambiente negativo para a cultura de segurança, mas não entendiam ainda seu papel como atores no processo (Carvalho \& Cassiani, 2012; Correggio et al., 2014).

Os resultados para percepção do estresse e das condições de trabalho também se apresentaram com clima negativo. Estudos apontam que a maior prevalência de estresse aumenta as chances de ocorrerem incidentes, assim como condições de trabalho inadequadas ocasionam estresse ocupacional com repercussão em um trabalho de alta exigência, influenciando negativamente os cuidados prestados ao paciente (Alves et al., 2004; Andolhe et al., 2015; Carvalho \& Cassiani, 2012). Assim, se o profissional não percebe o ambiente como estressante, também não percebe que isso prejudica o cuidado prestado.

Por fim, evidenciou-se que: quanto maior a despersonalização, menor é a percepção positiva da cultura de segurança; quanto maior a demanda psicológica, menor é a percepção positiva da cultura de segurança e quanto maior a realização profissional, maior é a percepção positiva da cultura de segurança. Em vista disso, constatou-se que profissionais de saúde com sindrome de Burnout e estresse ocupacional avaliam a cultura de segurança negativamente.

Destarte, uma vez que se tenha profissionais acometidos por Burnout ou estresse ocupacional, caso esses não utilizem estratégias de enfrentamento adequadas, haverá desacordos entre a pessoa e o seu ambiente de trabalho, pois esses profissionais encontram-se irritados, desmotivados, distanciados dos seus objetivos e com altas demandas psicológicas (Andolhe et al., 2015; Lautert, 1995; Munhoz et al., 2020). Assim, a cultura de segurança também poderá estar comprometida (Carvalho \& Cassiani, 2012).

Nesse contexto, a cultura de segurança está relacionada, dentre outros fatores, com o comportamento dos profissionais que atuam no ambiente de trabalho, esse que pode ser influenciado pelo estado de saúde (Carvalho \& Cassiani, 2012; Carvalho et al., 2016). Com isso, entende-se que os profissionais que estão em Burnout e em estresse ocupacional, encontram-se fragilizados emocionalmente, desgastados e com altas demandas psicológicas no trabalho, o que repercute em um profissional com pouco controle sobre o trabalho e que, consequentemente, avalia a cultura de segurança de forma negativa (Andolhe et al., 2015; Carvalho \& Cassiani, 2012; Correggio et al., 2014; Maslach, 2009).

Em suma, além do estresse ocupacional e do Burnout, denota-se que a complexidade dos pacientes e a rotina com altas demandas de tra- 
balho dos ambientes de perioperatório também fragilizam a percepção da cultura de segurança (Brandão \& Galvão, 2013; Gonçalves \& Medeiros, 2016). Porém, é indispensável que esses profissionais busquem ter mais subsídios para, assim, terem controle sobre suas demandas laborais e, com isso, favorecerem a cultura de segurança (Andolhe et al., 2015; Carvalho \& Cassiani, 2012). A exemplo disso, deve-se dar seguimento com as ações de ensino aprendizagem, desde que essas ações sejam avaliadas constantemente, com feedback a equipe. Dessa forma, será possivel constatar o que precisa mudar no ambiente de trabalho e o que pode ser mantido.

Ademais, outras ações podem ser feitas com vistas a favorecer a saúde do trabalhador, como: realização de intervenções para a identificação de estressores laborais e estratégias de enfrentamento; conscientização sobre prevenção de doenças ocupacionais e; estímulo ao trabalhador manter-se saudável. Em relação à cultura, sugerem-se estratégias que a favoreçam, como: o diálogo aberto e horizontal entre chefias e profissionais assistentes e incentivar os profissionais a participarem ativamente das atividades de educação em saúde voltadas à segurança do paciente.

\section{Conclusão}

Evidenciou-se que a população estudada tinha uma percepção negativa da cultura de segurança nas unidades de perioperatório. Também prevaleceu o número de profissionais de saúde em trabalho de alta exigência, com alto desgaste emocional, com alta despersonalização e com alta realização profissional. Outrossim, além da percepção acerca da cultura de segurança de unidades de perioperatório, foi possivel observar a presença de estresse do momento atual (estresse ocupacional) e crônico (Burnout) dos profissionais de saúde. Em vista disso, a hipótese deste estudo foi confirmada, pois constatou-se que a ocorrência de estresse ocupacional e Burnout possui correlação inversa e significativa com a cultura de segurança. Ou seja, profissionais de saúde acometidos por sindrome de Burnout e estresse ocupacional avaliam a cultura de segurança ne- gativamente. Esses resultados são importantes para a que gestores e gerentes possam planejar investimento em melhores condições de trabalho, com ambientes saudáveis para o trabalhador, a fim de melhorar a percepção da cultura de segurança. Investir na saúde do trabalhador pode representar investir na segurança do paciente.

Este estudo apresentou como limitação o viés da temporalidade, uma vez que não é possivel estabelecer relação de causa e efeito entre as variáveis estudadas. A partir dessa limitação, pesquisas futuras poderão explorar outras variáveis e utilizar delineamentos que permitam investigar intervenções voltadas tanto à saúde do trabalhador, quanto à segurança do paciente.

\section{Referências}

Agência Nacional de Vigilância Sanitária. (2013). Resolução da Diretoria Colegiada da ANVISA- RDC IAnvisa $n^{\circ} 36$, de 35 de julho de 2013]. Institui ações para a segurança do paciente em serviços de saúde e dá outras providências. Diário Oficial da União.

Alves, M. G. M., Chor, D., Faerstein, E., Lopes, C. S., \& Wernwck, G. L. (2004). Short version of the "job stress scale": a Portuguese-language adaptation. Revista Saúde Pública, 38(2). http://dx.doi.org/10.1590/S003489102004000200003

Amthauer, C., \& Falk, J. W. (2014). O enfermeiro no cuidado ao paciente cirúrgico no periodo pré-operatório. Revista Enf FW, 10(10), 54-59.

Andolhe, R., Barbosa, R. L., Oliveira, E. M., Costa, A. L. C., \& Padilha, K. G. (2015). Stress, coping and burnout among Intensive Care Unit nursing staff: associated factors. Revista Esc Enferm USP, 49(Esp.), 58-64. http:// dx. doi.org/10.1590/S0080-623420150000700009

Andrade, R. V. S., \& Costa, O. R. S. (2014). Estresse ocupacional em profissionais de saúde: um estudo com a equipe de enfermagem da unidade de terapia intensiva - UTI. Revista Ciências em Saúde, 4(4), 11. http://dx.doi. org/10.21876/rcsfmit.v4i4.261

Boas, A. C. V., Carmo, H. M. O., Silva, Q. R., Silveira, J., Meneguetti, D. U. O. (2016). Evaluation of the stress factors of the healthcare teams at the emergency of a unit reference of rio branco. Journal of Amazon Health Science, 2(3), 1-16.

Brandão, D. E. C., \& Galvão, C. M. (2013). O estresse da equipe de enfermagem que atua no periodo perioperatório: revisão integrativa. Revista Rene, 14(4), 836-44.

Carvalho, D., Silva, N., Bachur, J. A., Mesquita, J. L. F., \& França-Botelho, A. C. (2016). Burnout syndrome in health professionals acting on two municipalities of Minas Gerais - Brazil. Rev Contexto \& Saúde, 16(31), 140. https://doi.org/10.21527/2176-7114.2016.31.139-148 
Carvalho, R. E. F. L., Arruda, L. P., Nascimento, N. K. P., Sampaio, R. L., Cavalcante, M. L. S. N., \& Costa, A. C. P. (2017). Assessment of the culture of safety in public hospitals in Brazil. Rev Latino-Am Enfermagem, $25 \mathrm{e} 2849$. https://doi.org/10.15.90/1518-8345.1600.2849

Carvalho, R. E. F. L., \& Cassiani, S. H. B. (2012). CrosS-cultural adaptation of the Safety Attitudes Questionnaire - Short Form 2006 for Brazil. Rev Latino-Am Enfermagem, 20(3), 575-582. http://dx.doi.org/10.1590/ S0104-11692012000300020

Correggio, T. C., Amante, L. N., \& Barbosa, S. F. F. (2014). Evaluation of patient safety culture in Surgery Center. Revista SOBECC, 19(2), 67-73. http://dx.doi.org/10.4322/ sobecc.2014.012

Gonçalves, T. F., \& Medeiros, V. C. C. (2016). The preoperative visit as the anxiety mitigating factor in surgical patients. Revista SOBECC, 21(1), 22-27. https://doi. org/10.5327/Z1414-4425201600010004

Karasek, R., \& Theörell, T. (1990). Healthy work: stress, productivity, and the reconstruction of working life. Basic Books.

Lautert, L. (1995). O desgaste profissional do enfermeiro [Tese doutorado, Universidade Pontifícia de Salamanca].

Maslach, C. (2009). Comprendiendo el Burnout. Ciencia \& Trabajo, 11(32), 37-43.

Maslach, C., \& Jackson, S. (1986). Maslach Burnout Inventary, Manual. University of California. Consulting Psychologists.

Matiello, R. D. C., Lima, E. F. A., Coelho, M. C. R., Oliveira, E. R. A., Leite, F. M. C., \& Primo, C.C. (2016). Patient safety culture from the perspective of nurses. Cogitare Enferm, 21(esp), 1-9.

Munhoz, O. L., Arrial, T. S., Barlem, E. L., Dalmolin, G. L., Andolhe, R., \& Magnago, T. S. (2020). Occupational stress and burnout in health professionals of perioperative units. Acta Paul Enferm, eAPE20190261. http://dx.doi. org/10.37689/actaape/2020A00261

Munhoz, O. L., Andolhe, R., Magnago, T. S. B. S., Dalmolin, G. L., \& Pasa, T. S. (2018). Profile of patients and incidents in a surgical clinic unit. Rev enferm UFPE, 12(2), 416-23. https://doi.org/10.5205/1981-8963-v12i2a230 813p416-423-2018

Oliveria, B. L., Filha, M. de O., Monteiro, C., Pinheiro, R., \& Figueiredo Cunha, C. (2013). Estresse entre graduandos de enfermagem de uma universidade pública federal: um estudo epidemiológico. JMPHC, 3(2), 72-79. https:// doi.org/10.14295/jmphc.v3i2.141

Prieto, G., \& Muñiz, J. (2000). Un modelo para evaluar la calidad de los tests utilizados en España. Papeles del Psicólogo, 77, 65-72.

Romero, M. P., González, R. B., Calvo, M. S. R., \& Fachado, A. A. (2018). A segurança do paciente, qualidade do atendimento e ética dos sistemas de saúde. Rev Bioética, 26(3), 333-342. https://doi.org/10.1590/1983-80422018263252

Silva, E. F. L., \& Moura, M. L. C. (2014). Stress in nurse/ patient relations: integrative review. J Nurs UFPE, 8(7), 2140-2148. https://doi.org/10.5205/1981-8963-v8i7ag 894p2140-2148-2014
Souza, V. F. S., \& Araujo, T. C. C. F. (2015). Estresse ocupacional e resiliência entre profissionais de saúde. Psicologia: ciência e profissão, 35(3), 900-915. http:// dx.doi.org/10.1590/1982-370300452014

Zanatta, A. B., \& Lucca, S. B. (2015). Prevalence of Burnout syndrome in health professionals of an onco-hematological pediatric hospital. Rev Esc Enferm USP, 49(2), 253-260. https://doi.org/10.1590/S0080$\underline{623420150000200010}$

\section{Oclaris Lopes Munhoz}

Mestre em Enfermagem pela Universidade Federal de Santa Maria (UFSM), em Santa Maria, RS, Brasil; doutorando em Enfermagem pelo Programa de Pós-Graduação em Enfermagem da Universidade Federal de Santa Maria (UFSM), em Santa Maria (UFSM), RS, Brasil.

\section{Laura Prestes Moreira}

Enfermeira pela Universidade Federal de Santa Maria (UFSM), em Santa Maria, RS, Brasil; m. Mestranda em Enfermagem pelo Programa de Pós-Graduação em Enfermagem da Universidade Federal de Santa Maria (UFSM), em Santa Maria, RS, Brasil.

\section{Adriane Cristina Bernat Kolankiewicz}

Doutora em Ciências pela Universidade Federal de São Paulo (UNIFESP), em São Paulo, SP, Brasil; professora da Universidade Regional do Estado do Rio Grande do Sul (UNIJUI), em ljuí, RS, Brasil.

\section{Graziele de Lima Dalmolin}

Doutora em Enfermagem pela Universidade Federal de Rio Grande (FURG), em Rio Grande, RS, Brasil; professora da Universidade Federal de Santa Maria (UFSM), em Santa Maria, RS, Brasil.

\section{Tânia Solange Bosi de Souza Magnago}

Doutora em Enfermagem pela Universidade Federal de Rio de Janeiro (UFRJ), no Rio de Janeiro, RJ, Brasil: professora da Universidade Federal de Santa Maria (UFSM), em Santa Maria, RS, Brasil.

\section{Rafaela Andolhe}

Doutora em Enfermagem na Saúde do Adulo pela Universidade Federal de São Paulo (INIFESP), em São Paulo, SP, Brasil; professora da Universidade Federal de Santa Maria (UFSM), em Santa Maria, RS, Brasil. 
12/12 Psico, Porto Alegre, v. 52, n. 2, p. 1-12, abr.-jun. $2021 \mid$ e-36085

\section{Endereço para correspondência}

Oclaris Lopes Munhoz

Universidade Federal de Santa Maria

Centro de Ciências da Saúde

Avenida Roraima, 1000, Prédio 26, sala 1431

Camobi, 9705-900

Santa Maria, RS, Brasil

Os textos deste artigo foram revisados pela Poá Comunicação e submetidos para validação do(s) autor(es) antes da publicação. 